\title{
BAO Plate Archive Project
}

A. M. Mickaelian, K. S. Gigoyan, M. V. Gyulzadyan, G. M. Paronyan, H. V. Abrahamyan, H. R. Andreasyan, N. M. Azatyan, G. R. Kostandyan, A. L. Samsonyan, G. A. Mikayelyan, S. V. Farmanyan, V. L. Harutyunyan

NAS RA V. Ambartsumian Byurakan Astrophysical Observatory (BAO), Armenia E-mail:aregmick@yahoo.com

\begin{abstract}
We present the Byurakan Astrophysical Observatory (BAO) Plate Archive Project that is aimed at digitization, extraction and analysis of archival data and building an electronic database and interactive sky map. BAO Plate Archive consists of 37,500 photographic plates and films, obtained with $2.6 \mathrm{~m}$ telescope, $1 \mathrm{~m}$ and $0.5 \mathrm{~m}$ Schmidt telescopes and other smaller ones during 1947-1991. The famous Markarian Survey (or the First Byurakan Survey, FBS) 2000 plates were digitized in 2002-2005 and the Digitized FBS (DFBS, www.aras.am/Dfbs/dfbs.html) was created. New science projects have been conducted based on this low-dispersion spectroscopic material. Several other smaller digitization projects have been carried out as well, such as part of Second Byurakan Survey (SBS) plates, photographic chain plates in Coma, where the blazar ON 231 is located and $2.6 \mathrm{~m}$ film spectra of FBS Blue Stellar Objects. However, most of the plates and films are not digitized. In 2015, we have started a project on the whole BAO Plate Archive digitization, creation of electronic database and its scientific usage. Armenian Virtual Observatory (ArVO, www.aras.am/Arvo/arvo.htm) database will accommodate all new data. The project runs in collaboration with the Armenian Institute of Informatics and Automation Problems (IIAP) and will continues during 4 years in 2015-2018. The final result will be an Electronic Database and online Interactive Sky map to be used for further research projects. ArVO will provide all standards and tools for efficient usage of the scientific output and its integration in international databases.
\end{abstract}

Keywords: photographic plates - plate archives - digitization - astrometry - photometry - spectroscopy - databases - virtual observatories.

\section{Introduction}

The astronomical plate archives created on the basis of numerous observations at many observatories are the most important part of the astronomical observational heritage. The necessity of digitization of astronomical plates was emphasized and current progress in various national and international projects was given at Astroplate workshops (e. g. Osborn 2014; Hudec 2014; Kazantseva 2014; Nesci et al. 2014a; Stupka \& Benesova 2014). 
Byurakan Astrophysical Observatory (BAO) Plate Archive is one of the largest astronomical archives in the world and is considered to be BAO main observational treasure. It is the results of decades' hard work of Armenian astronomers and the work of BAO telescopes and other expensive equipment, as well as the results of their activities. Today BAO archive holds some 37,000 astronomical plates, films or other carriers of observational data. However, previous observational and informational registration methods currently do not make it available to wide range of scientists, and especially its usage for solution of new research problems. Digitization of BAO plates will be a significant contribution to the Wide-Field Plate Data Base (WFPDB) developed in Sofia, Bulgaria (Tsvetkov \& Tsvetkova 2012).

A project on Digitization of BAO Plate Archive and creation of BAO Interactive Astronomical Database (shortly BAO Plate Archive project, BAO PAP) has started in February 2015. It is aimed at preservation of BAO valuable observational material accumulated during 1947-1991, creation of full Database of all BAO observations, creation of BAO Interactive Sky Map with visualization of all observations and quick access to the data, development and accomplishment of new research projects based on the existing observational material, and integration of BAO observations into the international databases. A number of BAO young astronomers are involved in this project and it will last 3 years.

Project objectives are the preservation of BAO observational archive, preservation of scientific information contained in photographic plates and other careers, creation of opportunity of dissemination and wide usage of observational data, putting in correspondence of observational material to modern standards and usage methods, proposing new science projects and creation of possibility of their further accomplishment, and making BAO activities visible.

A short description of BAO Plate Archive was given by Mickaelian (2014) and more detailed paper is given in Mickaelian et al. (2016b).

\section{BAO telescopes and observing programmes}

BAO observers worked with a number of BAO telescopes during 1947-1991 and obtained several dozens of thousands of plates, films and other products. The table gives general understanding on observations of 10 BAO telescopes that worked on photographic photometry, electrophotometry, slit and objective prism spectroscopy, and polarimetry of many thousands astronomical objects. 
Table 1. Overview of BAO telescopes and produced observational material

\begin{tabular}{|l|c|c|l|c|}
\hline \hline \multicolumn{1}{|c|}{ Telescope } & Sizes $(\mathrm{cm})$ & Years & $\begin{array}{c}\text { Observing } \\
\text { methods }\end{array}$ & Plates \\
\hline 5" double-astrograph & 13 & $1947-1950$ & photometry & 3000 \\
6" & 15 & $1947-1950$ & photometry & 3000 \\
8" Schmidt & $20 / 20 / 31$ & $1949-1968$ & photometry & 4500 \\
20" Cassegrain & $51 / 800$ & $1952-1991$ & electrophotometry & \\
10" telescope-spectrograph & 25 & $1953-19 ? ?$ & spectra & \\
Nebular spectrograph & & $1954-19 ? ?$ & spectra & \\
16" Cassegrain & $41 / 400$ & $1955-1991$ & electrophotometry & \\
21" Schmidt & $53 / 53 / 183$ & $1955-1991$ & photometry & 12000 \\
40" Schmidt (AZT-10) & $102 / 132 / 213$ & $1960-1991$ & photometry, spectra & 7500 \\
ZTA-2.6m & $264 / 1016$ & $1975-1991$ & photometry, spectra & 7000 \\
All telescopes & & $\mathbf{1 9 4 7 - 1 9 9 1}$ & & $\mathbf{3 7 0 0 0}$ \\
\hline
\end{tabular}

We give in Table 1 an overview of BAO telescopes and produced observational material. Telescope "Sizes" are given for the mirror and focal length for classical telescopes and for the correcting lens, mirror and focal length for Schmidt type telescopes. Here we list the main observational projects accomplished on the three most important BAO telescopes $(2.6 \mathrm{~m}$ classical reflector, $1 \mathrm{~m}$ Schmidt and $0.5 \mathrm{~m}$ Schmidt; Figure 1).

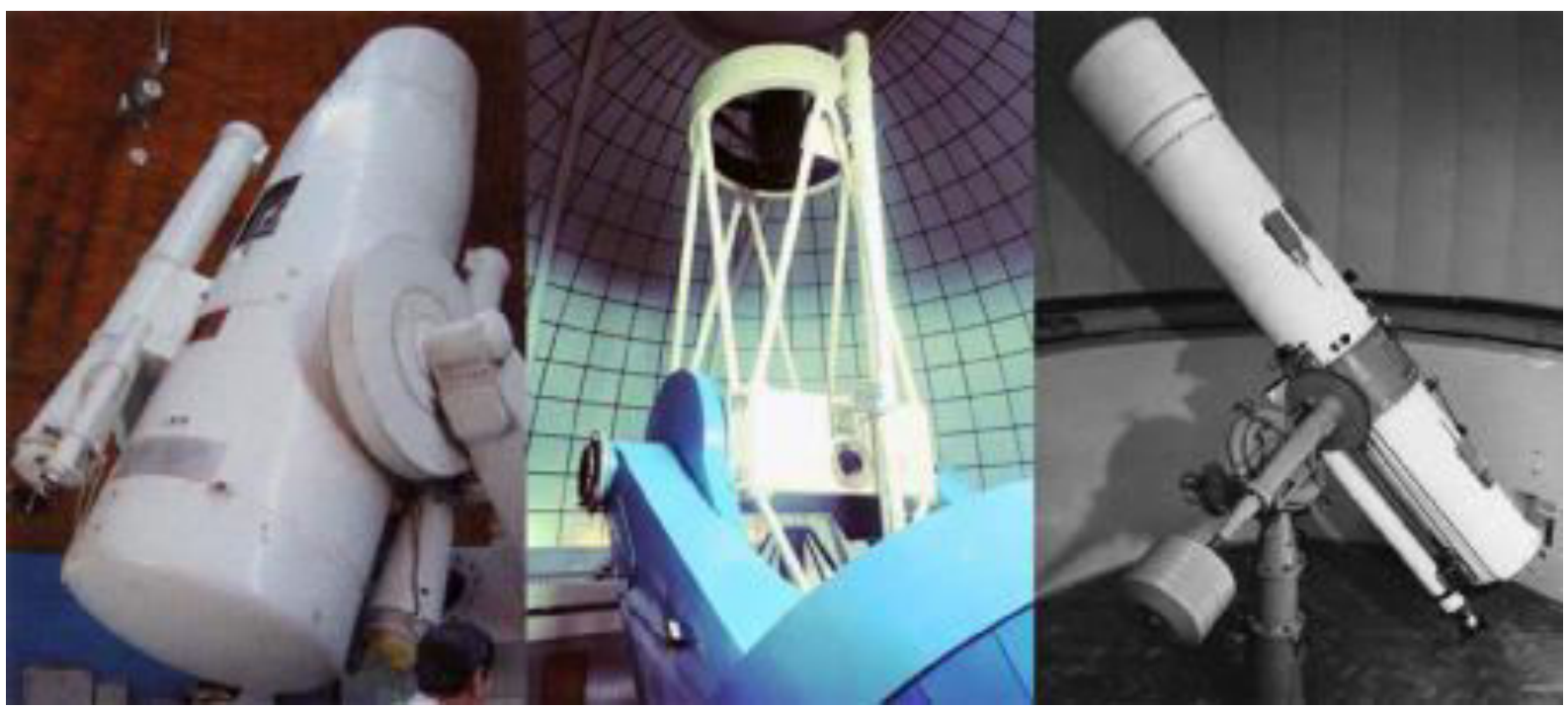

Figure 1. BAO most important telescopes (from left to right): $1 \mathrm{~m}$ Schmidt, $2.6 \mathrm{~m}$ classical reflector and $0.5 \mathrm{~m}$ Schmidt.

\section{1" (0.5m) Schmidt telescope:}

- Polarization of cometary nebula NGC 2261

- Nuclei of nearby Sa and Sb galaxies

- Nuclei of nearby Sc galaxies

- Search for flare stars in Pleiades

- Search for flare stars in Orion 
- Search for flare stars in NGC 7000 (Cygnus)

- Search for flare stars in Praesepe

- Search for flare stars in Taurus Dark Clouds (TDC)

- Variability of Markarian galaxies

- Monitoring of extragalactic supernovae in certain areas

\section{0" (1m) Schmidt telescope:}

- Detailed colorimetry of bright galaxies

- First Byurakan Survey (FBS, Markarian survey; Markarian 1989)

- Search for flare stars in Pleiades

- Search for flare stars in Orion

- Search for flare stars in NGC 7000 (Cygnus)

- Search for flare stars in Praesepe

- Search for flare stars in Taurus Dark Clouds (TDC)

- Second Byurakan Survey (SBS; Stepanian 2005)

- Extension of the FBS in the Galactic Plane

\section{ZTA-2.6m telescope:}

- Morphological study of Markarian galaxies

- Investigation of star clusters

- Investigation of groups and clusters of galaxies

- Spectroscopy FBS blue stellar objects

- Spectroscopy FBS late-type stars

- Spectroscopy SBS galaxies and stellar objects (BAO/SAO)

- Direct images of the central regions of Markarian galaxies

- Spectroscopy of T Tauri and flare stars

- Spectroscopy of Byurakan-IRAS Galaxies (BIG objects)

- Spectroscopy of ROSAT AGN candidates (BAO/HS/OHP/INAOE)

Summarizing, the main observational projects run on these telescopes were: 21" (0.5m) Schmidt: Polarization of cometary nebula NGC 2261, Nuclei of nearby Sa and Sb galaxies, Nuclei of nearby Sc galaxies, Search for flare stars in Pleiades, Orion, NGC 7000 (Cygnus), Praesepe and Taurus Dark Clouds (TDC), Variability of Markarian galaxies, Monitoring of extragalactic supernovae in certain areas, etc.; 40" (1m) Schmidt: First Byurakan Survey (FBS, Markarian survey; Markarian 1989), Second Byurakan Survey (SBS; Stepanian 2005), Extension of the FBS in the Galactic Plane, Detailed colorimetry of bright galaxies, Search for flare stars in Pleiades, Orion, NGC 7000 (Cygnus), Praesepe and Taurus Dark Clouds (TDC), etc.; and ZTA-2.6m 
telescope: Morphological study of Markarian galaxies, Investigation of star clusters, Investigation of groups and clusters of galaxies, Spectroscopy of FBS blue stellar objects, FBS late-type stars, SBS galaxies and stellar objects (BAO/SAO), T Tauri and flare stars, Byurakan-IRAS Galaxies (BIG objects) and ROSAT AGN candidates (BAO/HS/OHP/INAOE), and Direct images of the central regions of Markarian galaxies. Especially efficient were Byurakan surveys accomplished by Markarian and colleagues: FBS and SBS.

Scientific Programs Board (SPB) is created to evaluate the existing observational material, to select sets of priorities to be scanned first and to propose new research projects. It consists of BAO Director, DFBS Principal Investigator and ArVO Project Manager Areg Mickaelian (Chair), the Head of BAO Astroinformatics Department Tigran Magakian and 7 other most experienced BAO observers, as well as researchers from NAS RA Institute of Informatics and Automation Problems (IIAP) Vladimir Sahakian and Hrachya Astsatryan are involved for their experience in computer science related to databases and computational methods.

Project Executing Team (PET) consists of 14 members: Kamo Gigoyan (Project Manager, SPB member), Marietta Gyulzadyan (Deputy Manager on Observing Programs), Gurgen Paronyan (Deputy Manager on Technical Issues, Person in charge for BAO Plate Archive), 6 other PET members involved in scanning and reduction of data, Gor Mikayelyan (Database Manager, Web Designer), Sona Farmanyan (Webpage content, dissemination, outreach, and organizational issues) and Aram Knyazyan (NAS RA IIAP, Database Manager).

The project consists of the following tasks:

- Development of technical principles of the Project, necessary Equipment, Timeline and the Budget

- Collection of all photographic plates (until recently only plates obtained before 1974 had been collected in BAO Plate Archive)

- Revision and accounting of the plates and observing journals in BAO Plate Archive,

- Scanning of a few dozens of plates for test and educational reasons to set up the necessary parameters for the scanning in frame of the main Project

- Input of data from observing journals; Creation of the Project Database and development of the principles of organization of data in it

- Creation of the Project Webpage and User Interface 
- Scanning of photographic plates and films

- Astrometric solution; Extraction of images and spectra; Wavelength calibration; Density and flux calibration; Multiband (UBVR) photometry

- Making up template low-dispersion spectra; Numerical classification of low-dispersion spectra

- Visualization of BAO observations on sky map; Creation of electronic interactive sky map and search system

- Scientific analysis of existing observational material and providing new research possibilities; Proposing and discussing new research projects

BAO PAP webpage (http://www.aras.am/PlateArchive/; Figure 2) was recently open and contains a lot of information on BAO observations, previous digitization projects, present Project details, teams, follow-up research projects, deliverables and related links (many items will be filled in during the next months). The main products will be "Data Access" and "Interactive Sky Map". The first one will contain BAO Observational Database, Search by any parameter (Dates / Julian dates, Telescope, Observing modes/methods, Instrument, Receiver, Emulsion, Filters, Seeing, Project name, Project PI, Observers, Targets / coordinates, Sky area, Surface, Scale, Spatial resolution, Spectral range, Spectral resolution, Limiting magnitude, Number of nights, Number of exposures, Links), Data Visualization and Download of the digitized plates, films, part of them or individual objects images or spectra. "Interactive Sky Map" will visualize the observed by BAO telescopes sky and will give possibility to check observed areas for a given observational project, given telescope, observer, observing method, limiting magnitude, etc. There will be possibility to check individual fields for presence and number of plates to propose further research projects. Main expected projects are supposed to be those on variability and proper motions, as well as studies of the Solar System objects.

\section{DFBS and other digitization projects at BAO}

A number of digitization projects have been accomplished at $\mathrm{BAO}$, including the most important one, Digitized First Byurakan Survey (DFBS; http://www.aras.am/Dfbs/dfbs.html; Mickaelian et al. 2007; Massaro et al. 2008) based on the digitization of the famous Markarian Survey (Markarian et al. 1989). Its main scanning and resulting features are given in Table 2. 


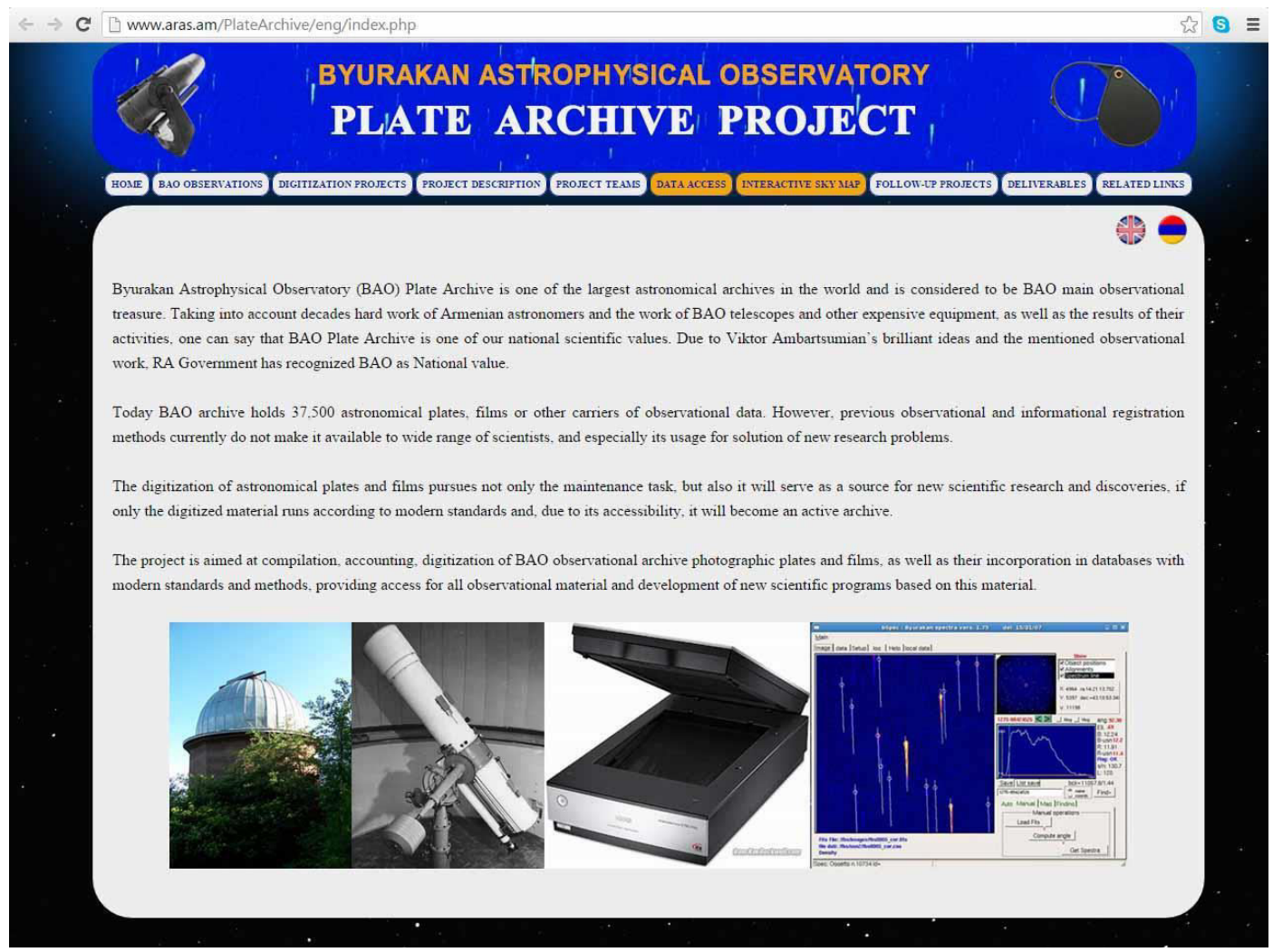

Figure 2. BAO Plate Archive Project webpage.

Table 2. Main scanning and resulting characteristics of the DFBS

\begin{tabular}{|l|l|}
\hline Items & Description \\
\hline Teams & Byurakan Astrophys. Obs., Univ. Roma "La Sapienza", Cornell Univ. \\
Years & 2002-2005 \\
Instrument & Epson Expression 1680 Pro scanner \\
Scanning options & 1600 dpi $(15.875 \mu$ pix size), 16 bit, transparency mode, "scanfits" \\
Plate size & $9601 \times 9601$ pix, 176 MB file \\
Spectra & $107 \times 5$ pix $(1700 \mu$ in length) \\
Dispersion & $33 \AA /$ pix average $(22-60 \AA /$ pix $), 28.5$ at $\mathrm{H} \gamma$ \\
Spectral resolution & $50 \AA$ (average) \\
Astrometric solution & $1 "$ rms accuracy \\
Scale & $1.542 " /$ pix \\
Photometry & $0.3^{m}$ accuracy \\
Data volume & 1874 plates, $\sim 400 \mathrm{~GB}$ \\
Number of objects & $\sim 20,000,000(\sim 40,000,000$ spectra) \\
\hline
\end{tabular}

We give in Figure 3 (left panel) a piece of visualized DFBS field together with its corresponding DSS2 area. Some 40,000,000 DFBS low-dispersion spectra have been extracted from 1874 plates, measured and analyzed by means of the dedicated software bSpec (Figure 3, right panel), written by Giuseppe Cirimele.

The spectra extraction and analysis software is described in Mickaelian et al. (2010) and Knyazyan et al. (2011). DFBS plate database is available in Vizier, Strasbourg (Mickaelian et al. 2005). 

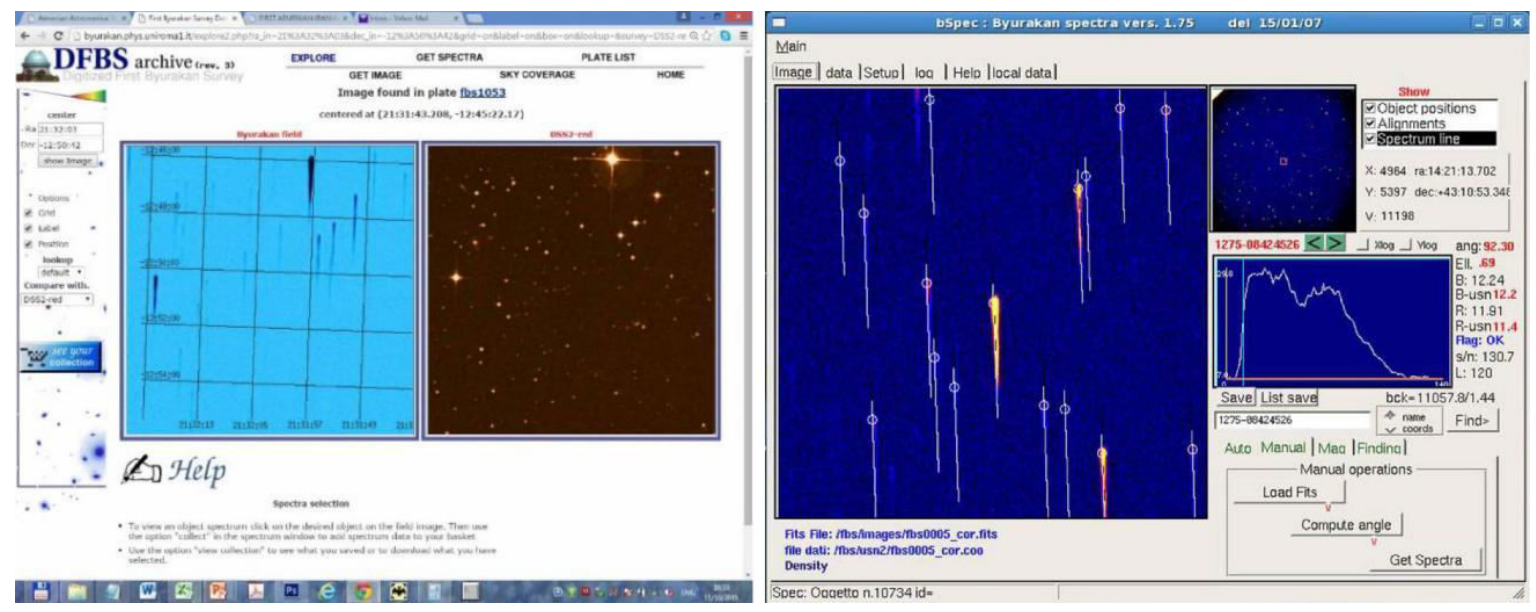

Figure 3. Left: DFBS data visualization with comparison of similar DSS2 field. Right: DFBS spectra extraction and analysis software bSpec.

In Figure 4 we give an example of extraction of an asteroid spectrum from DFBS using VO software SkyBoT proving how useful the DFBS plates can be for follow-up studies (Thuillot et al. 2007; Berthier et al. 2009; Sarkissian et al. 2012). The search for asteroids in DFBS jointly with IMCEE (Observatoire de Paris, France) colleagues was the most advanced research project. Bright $\left(<15^{\mathrm{m}}-16^{\mathrm{m}}\right)$ asteroids observed in DFBS are being studied, which are divided into "fast" and "slow" ones depending on their motion during the typical DFBS plate exposure time (20 min), more or less than 3 . All asteroid spectra are being extracted after they are found by means of SkyBoT. Sample spectra are being modelled similar to Solar spectra. Using these spectra and by means of comparisons with other catalogues, new candidate asteroids are being searched. Spectra analysis of asteroid spectra is being accomplished aimed at obtaining definite physical parameters.

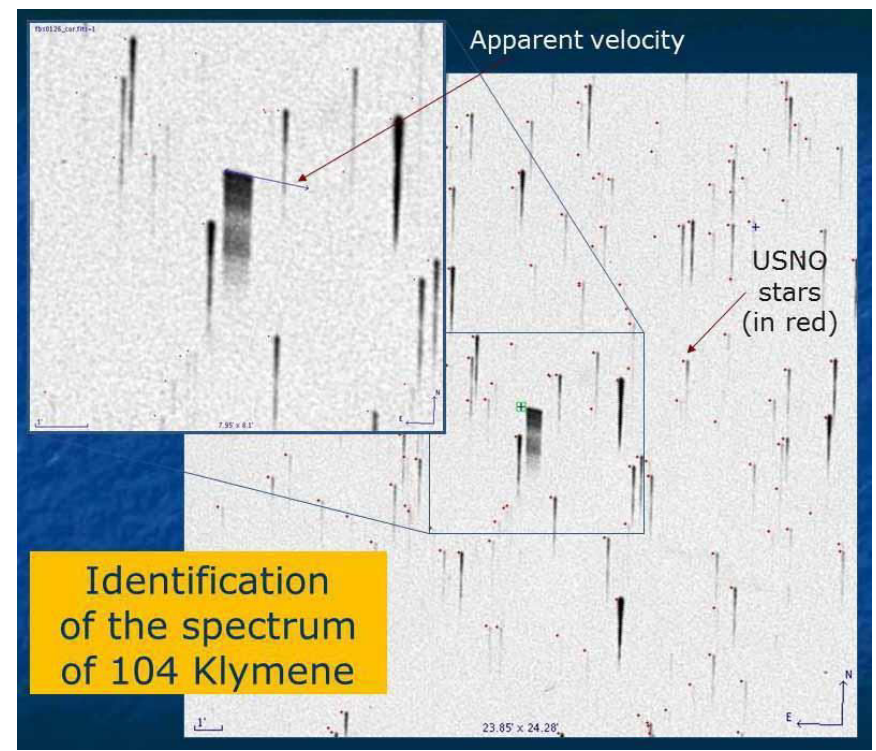

Figure 4. Extraction of an asteroid spectrum from DFBS using VO software SkyBoT. 


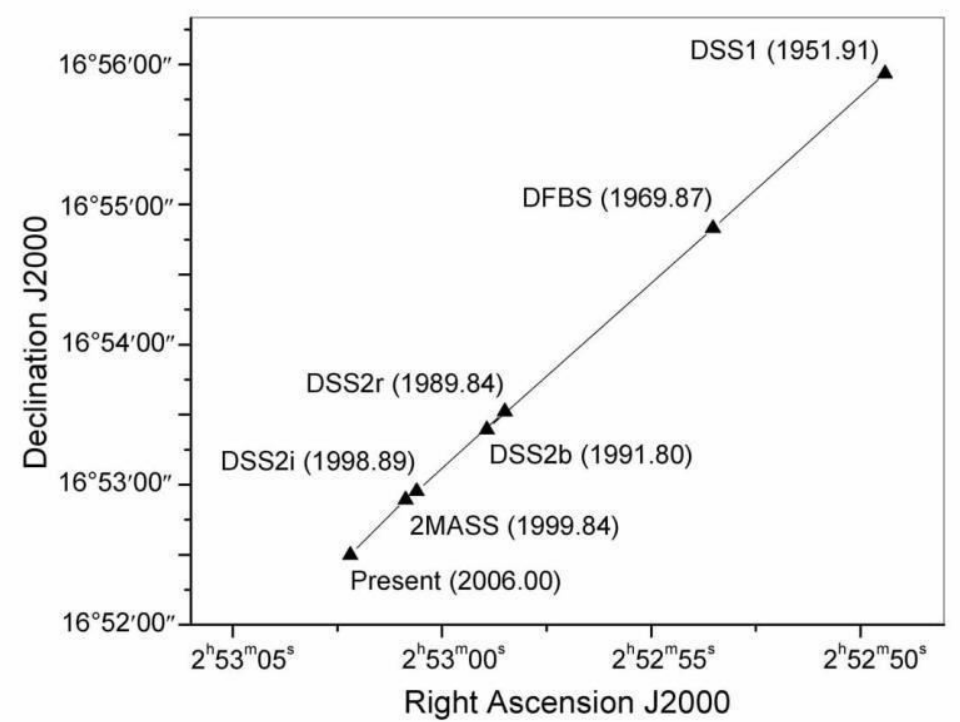

Figure 5. Direction of the motion of the high proper motion (PM=5.050/yr) star FBS $0250+167$, which was found due to DFBS plates.

The efficiency of studies of AGB stars based on DFBS low dispersion spectra and follow-up spectroscopy was shown by Nesci et al. (2014b). Gigoyan \& Mickaelian (2007) have found a very high proper motion (PM) M7 type dwarf star, lying about 3 pc from the Sun, FBS $0250+167$. Its PM is $5.050 / y r$ and it is in the list of the 10 known highest PM stars. Figure 5 shows that only existence of additional observational material, namely DFBS plates from 1969, linked measurements between DSS1 and DSS2 and helped identifying this object and measuring its PM.

The Second Byurakan Survey (SBS; Stepanian 2005) plates are also subject for digitization, as they are hypersensitized and their emulsion is more sensitive for deterioration. 180 plates have been digitized so far. Due to SBS smaller photographic grains, $2400 \mathrm{dpi}(10 \mu \mathrm{m}$ pixel size) is being used and $512 \mathrm{MB}$ files are being obtained for each plate.

Photographic spectra of the FBS blue stellar objects (BSOs) have been obtained using 2.6m telescope and UAGS spectrograph on photographic films. 700 such spectra have been scanned with $1600 \mathrm{dpi} 16$ bit and 65021 pix sizes images were obtained (FBS BSOs; Mickaelian 2008 and late-type stars; Gigoyan \& Mickaelian 2012). All spectra were put in a standard format, so that automatic reduction was possible (Figure 6). 101 FBS blue stellar objects were published and a number of planetary nebulae, white dwarfs, hot subdwarfs and HBB stars have been revealed (Sinamyan \& Mickaelian 2009).

Another project was the study of long-term variability of ON 231, which appeared in the Coma field, where photographic chains for discovery of flare stars were carried out. In total 189 plates with a total number of more than 1200 exposures in 1969-1976 with the Byurakan 21" and 40" Schmidt telescopes were 
obtained. This was a valuable material for study of ON231 long-term variability (Figure 7; Erastova 2004).

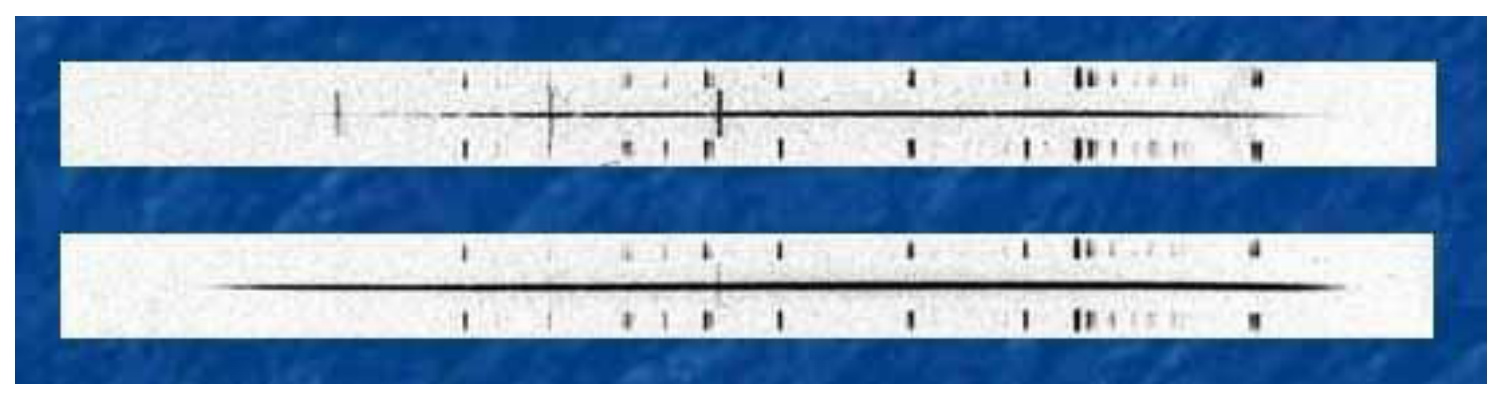

Figure 6. Standard format of FBS spectra with 65021 pix sizes images for automatic reduction.

Having digitized plates and modern digital observational data, a number of efficient research projects have become possible, such as data discovery, spectral analysis, SED building and fitting, modelling, variability studies, cross-matching, etc. Some examples are variability studies (Samus \& Antipin 2012), Cross-matching of Astronomical Catalogs (Malkov 2012), Search for Asteroids and Exoplanets using VO tools (Sarkissian et al. 2012).
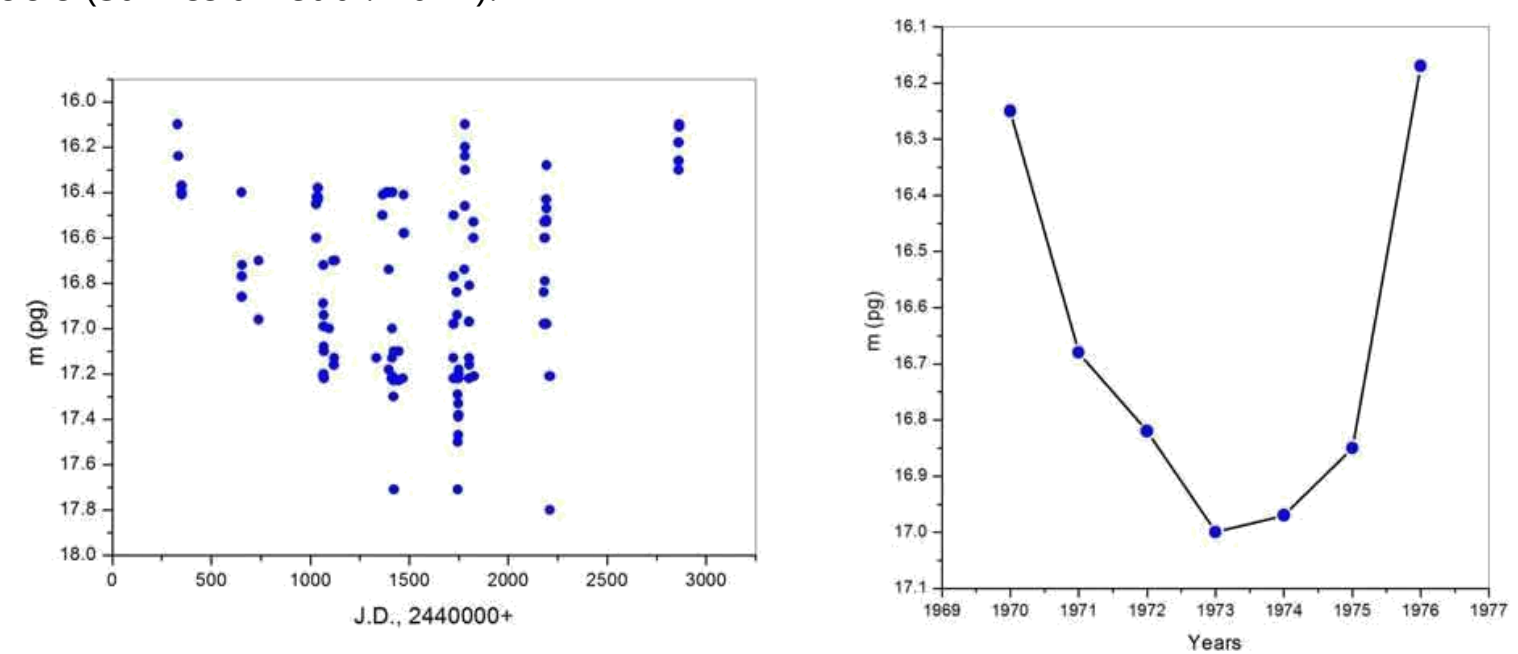

Figure 7. Photometric measurements and variability study of the blazar ON 231 observed in the Coma field.

Our science projects are aimed at discoveries of new interesting objects searching definite types of low-dispersion spectra in the DFBS, by optical identifications of non-optical sources (X-ray, IR, radio) also using the DFBS and DSS/SDSS, by using cross-correlations of large catalogs and selection of objects by definite criteria, etc.

In one of our papers (Abrahamyan et al. 2012), a new tool for cross-correlation of catalogs is presented (Knyazyan et al. 2011), where individual positional error for each object instead of a standard search radius is being taken into account, thus making the result much more accurate and confident (Figure 8). 


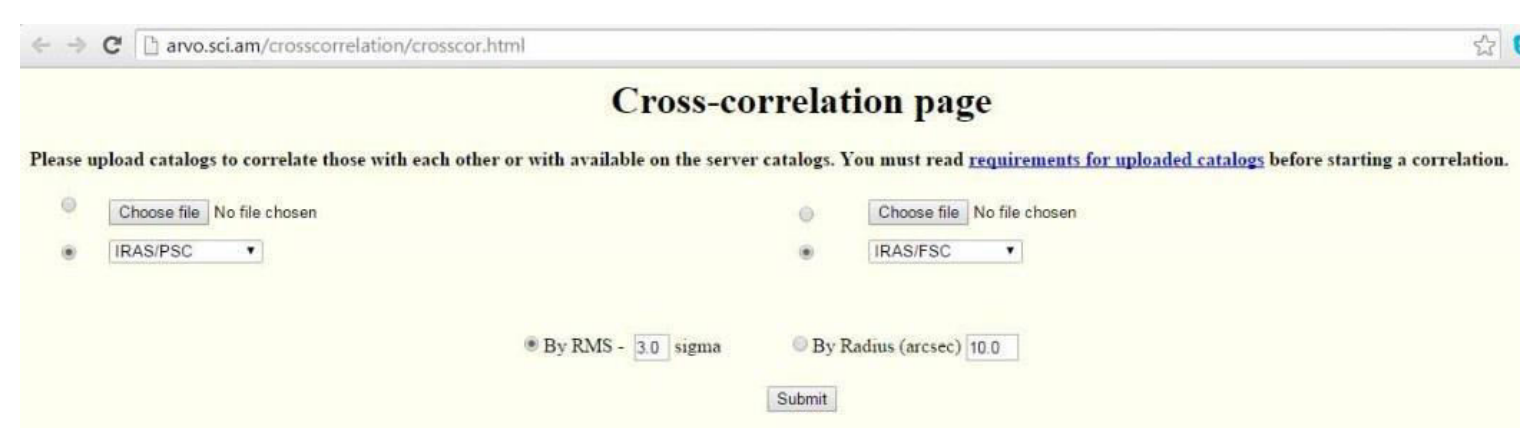

Figure 8. Cross-correlation software developed in frame of ArVO.

\section{Summary}

At present the main part of the project, the scanning of the plates has produced more than 15,000 digital images of roughly $200 \mathrm{MB}$ files (each image). All they have been stored and double copies are available.

BAO Plate Archive Project will lead to preservation of BAO valuable observational material obtained during 1947-1991. However, our goal is not only to create a passive archive of scanned plates and films, but also to make use of especially those fields, where more studies are possible.

Proper motion and variability studies are most important, as time domain material is contained in historical plates. Such possibilities based on DFBS were shown by Mickaelian et al. (2006); DFBS as a unique database for proper motion, variability studies, and object classification. New variable stars discovered on digitized plates of Moscow collection was reported by Sokolovsky et al. (2014).

There are a number of further possible research projects that will be conducted having the plates digitized:

- Correction of ephemerides of known asteroids and search for new asteroids (ex. Thuillot et al. 2007; Berthier et al. 2009)

- Discovery and study of variable stars (ex. Mickaelian et al. 2011; Nesci et al. 2009)

- Revealing high proper motion stars (ex. Mickaelian \& Sinamyan 2010)

- Study of variability of known blazars and discovery of new blazars

- Revealing Novae and Supernovae progenitors

- Discovery of new QSOs

- Discovery of new white dwarfs (ex. Sinamyan \& Mickaelian 2011)

- Discovery of new late-type stars (ex. Gigoyan et al. 2010)

- Discovery of optical sources of gamma-ray bursts 
- Optical identifications of X-ray, IR and radio sources (ex. Mickaelian \& Sargsyan 2004; Mickaelian \& Gigoyan 2006; Mickaelian et al. 2006; Hovhannisyan et al. 2009).

Acknowledgments. BAO Plate Archive Project team would like to thank BAO Administration for supporting the project and Scientific Programs Board (SPB) members for useful discussions on possible research programmes based on BAO Plate Archive material.

\section{References}

Berthier, J.; Sarkissian A.; Mickaelian A.; Thuillot W. 2009, Eur. Planetary Sci. Congress, Vol. 4, 526 Erastova, L. K. 2004, A\&AT 23, 209

Gigoyan, K. S.; Mickaelian A. M. 2007, Astrophysics 50, 54

Gigoyan K. S.; Mickaelian A. M. 2012, MNRAS 419, 3346

Gigoyan, K. S.; Sinamyan P. K., Engels D., Mickaelian A. M. 2010, Astrophysics 53, 123 Hovhannisyan, L. R.; Weedman D. W., Mickaelian A. M., et al. 2009, AJ 138, 251 Hudec, R. 2014, Proc. Astroplate-2014 Conf., Eds. L. Mišková \& S. Vítek. Prague, p. 1 Kazantseva, L. 2014, Proc. Astroplate-2014 Conf., Eds. L. Mišková \& S. Vítek. Prague, p. 13

Knyazyan, A.; Mickaelian A. M.; Astsatryan H. 2011, Int. J. "Inform. Theories and Appl." 18,243

Knyazyan, A. V.; Astsatryan, H. V.; Mickaelian, A. M. 2016, Astronomical Surveys and Big Data, Eds. A. M. Mickaelian, A. Lawrence and T. Yu. Magakian. ASP Conf. Series, Vol. 505, p. 236

Markarian, B. E.; Lipovetsky, V. A.; Stepanian, J. A.; et al. 1989, Comm. SAO 62, 5 Massaro, E.; Mickaelian A.M.; Nesci R.; Weedman D. (Eds.) 2008, The Digitized First Byurakan Survey, ARACNE Editrice, Rome, 78p.

Mickaelian, A. M. 2006, Rom. Astron. J. 16S, 23 Mickaelian, A. M. 2007, Highlights of Astronomy 14, 594

Mickaelian, A. M. 2008, AJ 136, 946

Mickaelian, A. M. 2012, Proc. Conf. "50 years of Cosmic Era: Real and Virtual Studies of the Sky". Yerevan, NAS RA, p. 29

Mickaelian, A. M. 2014, Proc. Astroplate-2014 Conf., Eds. L. Mišková \& S. Vítek.

Prague, p. 109 Mickaelian, A. M., Astsatryan, H. V., Sahakyan, V. G., et al. 2009, Proc. CSIT-2009, p. 420 Mickaelian, A. M., Gigoyan, K. S. 2006, A\&A 455, 765

Mickaelian, A. M.; Gigoyan, K. S.; Nesci, R.; Rossi, C. 2006, Mem.S.A.It. 77, 1159 Mickaelian, A. M.; Hagen, H.-J.; Sargsyan, L. A.; Mikayelyan, G. A. 2005, Catalog No. $\mathrm{VI} / 116$ at $\mathrm{CDS}$, Strasbourg 
Mickaelian, A. M.; Hovhannisyan, L. R.; Engels, D., et al. 2006, A\&A 449, 425 Mickaelian, A. M.; Kochiashvili N.; Astsatryan H. V., et al. 2009, Proc. CSIT-2009, p. 424 Mickaelian, A. M.; Malkov O. Yu.; Samus N. N. (Eds.) 2012, Proc. Conf. 50 years of Cosmic Era: Real and Virtual Studies of the Sky. NAS RA, 251 p., Yerevan Mickaelian, A. M.; Mikayelyan G.A.; Sinamyan P.K. 2011, MNRAS 415, 1061 Mickaelian, A. M.; Nesci R.; Cirimele G., et al. 2008, Proc. ESAC Workshop on Astronomical Spectroscopy and Virtual Observatory, Madrid, ESA, p. 29 Mickaelian, A. M.; Nesci, R.; Rossi C., et al. 2007, A\&A 464, 1177 Mickaelian, A. M.; Sargsyan L. A.; Nesci R., et al. 2010, ASP Conf. Series, Vol. 434: Astronomical Data Analysis Software and Systems XIX, p. 325 Mickaelian, A. M.; Sargsyan, L. A. 2004, Astrophysics 47, 213 Mickaelian, A. M.; Sargsyan, L. A.; Astsatryan H. V., et al. 2009, Data Science Journal 8, 152

Mickaelian, A. M.; Sargsyan, L. A.; Gigoyan K. S., et al. 2009, Rom. Astron. J. 18S, 249 Mickaelian, A. M.; Sargsyan, L. A.; Mikayelyan G. A. 2010, Proc. of Science, 30 Mickaelian, A. M.; Sargsyan, L. A.; Mikayelyan G. A., et al. 2006, Heron Press Sci. Ser., p. 82

Mickaelian, A. M.; Sargsyan, L. A.; Nesci R., et al. 2007, Highlights of Astronomy 14 Mickaelian, A. M.; Abrahamyan, H. V.; Andreasyan, H. R.; et al. 2016b, Astronomical Surveys and Big Data, Eds. A. M. Mickaelian, A. Lawrence \& T. Yu. Magakian. ASP Conf. Ser., V. 505, p. 262 Mickaelian, A., Sinamyan P. 2010, MNRAS 407, 681 Mickaelian, A. M. 2014, Multiwavelength AGN Surveys and Studies, Proc. IAU S304, Vol. 304, p.1

Mickaelian, A. M.; Astsatryan, H. V.; Knyazyan, A. V.; et al. 2016a, Astronomical Surveys and Big Data, Eds. A. M. Mickaelian, A. Lawrence and T. Yu. Magakian. ASP Conf. Ser., Vol. 505, p. 16

Nesci, R.; Bagaglia M.; Nucciarelli G. 2014a, Proc. Astroplate-2014 Conf., Eds. L. Mišková \& S. Vítek. Prague, p. 75

Nesci, R.; Gaudenzi S.; Rossi C.; Pezzotti C.; Gigoyan K., Mauron N. 2014b, Proc. Astroplate-2014 Conf., Eds. L. Mišková \& S. Vítek. Prague, p. 91

Nesci, R.; Mickaelian A. M.; Rossi C. 2009, Astron. Tel. \#2338 Osborn, W. 2014, Proc. Astroplate-2014 Conf., Eds. L. Mišková \& S. Vítek. Prague, p. 15

Sarkissian, A.; Arzoumanian E; Mickaelian A. M., et al. 2012, Proc. Conf. "50 years of Cosmic Era: Real and Virtual Studies of the Sky". Yerevan, NAS RA, p. 56

Sinamyan, P. K.; Mickaelian A. M. 2009, Astrophysics 52, 76

Sinamyan, P. K.; Mickaelian A. M. 2011, Astrophysics 54, 403

Sokolovsky, K. V.; Antipin S. V.; Zubareva A. M., et al. 2014, ARep 58, 319 
Stepanian, J.A. 2005, RMxAA 41, 155

Stupka, J.; Benesova E. 2014, Proc. Astroplate-2014 Conf., Eds. L. Mišková \& S. Vítek.

Prague, p. 31 Thuillot, W., Berthier J., Sarkissian A., et al. 2007, Highlights of

Astronomy 14, 616

Tsvetkov, M.; Tsvetkova, K. 2012, Proc. IAU S285: New Horizons in Time-Domain

Astronomy, Cambridge Univ. Press, p. 417

Véron-Cetty, M.-P.; Véron, P. 2010, A\&A 518, 10 